\title{
Reliability of Electrostatically Actuated RF MEMS Switches
}

\author{
James C. M. Hwang \\ Lehigh University, Bethlehem, Pennsylvania 18015 USA
}

\begin{abstract}
The reliability of electrostatically actuated RF MEMS switches is reviewed with emphasis on recent advancements. Both ohmic and capacitive switches have been operated for more 100-billion cycles without failure. When they do fail, ohmic switches tend to fail catastrophically by stiction, whereas capacitive switches often degrade gradually through charging of their dielectric insulators. Approaches to mitigate and model dielectric charging are then presented.
\end{abstract}

Index Terms - Charge injection, contacts, dielectric films, dielectric materials, microelectromechanical devices, ohmic contacts, switches.

\section{INTRODUCTION}

Micro-electro-mechanical systems (MEMS) devices can be divided into three classes: The critical mechanical elements of the first class of MEMS devices (e. g., accelerometers) move but rarely touch another element. The critical elements of the second class of MEMS devices (e. g., switches) make intermittent contact with another element. The critical elements of the third class of MEMS devices (e. g., gears) are in constant contact with another element. The first class of MEMS devices have proven to be so reliable that our lives can depend on them as in the case of airbag triggers. The third class of MEMS devices still suffers from infant mortality. The second class of MEMS devices are becoming reliable and are the present focus.

Switches, especially electrostatically actuated switches, are relatively simple to design, fabricate, and operate. They are not only basic building blocks of highly integrated RF MEMS, but also ideal vehicles to investigate the reliability of the second class of MEMS devices. There are two major types of switches: ohmic and capacitive. Ohmic switches make direct metal-tometal contacts while capacitive switches make metalinsulator-metal contacts. Both ohmic and capacitive switches have been operated for more than 100-billion cycles without failure [1], [2]. While the mechanisms for their eventual failure are still being investigated, their failure modes are relatively well established. Ohmic switches usually fail catastrophically by stiction, whereas capacitive switches often degrade gradually through charging of their dielectric insulators. In addition, dielectric charging is reversible so failed capacitive switches can often be operated again after resting overnight, especially at elevated temperatures.
TABLE I

Popular SElECtion OF CONTACT Metals

\begin{tabular}{|c|c|c|c|c|}
\hline Metal & $\begin{array}{c}\text { Resistivity } \\
\left(10^{6} \Omega-\mathrm{cm}\right)\end{array}$ & $\begin{array}{c}\text { Hardness } \\
(\mathrm{Mpa})\end{array}$ & $\begin{array}{c}\text { Melting } \\
\text { Pt. }\left({ }^{\circ} \mathrm{C}\right)\end{array}$ & $\begin{array}{c}\text { Chemical } \\
\text { Reactivity }\end{array}$ \\
\hline $\mathrm{Au}$ & 2 & 18 & 1060 & Lowest \\
\hline $\mathrm{AuNi}$ & 12 & 12 & 1040 & Low \\
\hline $\mathrm{Rh}$ & 4 & 12 & 1960 & Medium \\
\hline $\mathrm{Ru}$ & 7 & 9 & 2330 & Medium \\
\hline $\mathrm{In}$ & 5 & 9 & 2460 & Medium \\
\hline $\mathrm{W}$ & 5 & 10 & 3420 & High \\
\hline $\mathrm{Mo}$ & 5 & 9 & 2620 & High \\
\hline
\end{tabular}

\section{FAILURE MECHANISMS OF OHMIC SWITCHES}

Similar to conventional micro-relays, MEMS ohmic switches can fail due to either mechanical or electrical stress [3]. Mechanical stress can cause deformation, fatigue, and wear. Electrical stress can cause arcing, melting, welding, and electro-migration. One or more of these mechanisms in combination can in turn cause material to be transferred and surface film to be formed or ruptured. Contact resistance can then increase or decrease. Without detailed material characterization, it is impossible to ascertain which mechanism is responsible for the change in contact resistance, let alone to predict which mechanism will limit the lifetime of a particular switch design. The situation is exasperated by the low contact force $(\sim 100 \mu \mathrm{N})$ and high surface-to-volume ratio of MEMS, making ohmic switches particularly vulnerable to surface contamination.

Still, general rules can be developed to predict which failure mechanisms are more likely to be operating for a particular switch design. For example, the selection of the contact metal is the most critical factor in determining the reliability of ohmic switches. Table I lists the popular choices of contact metals, ranging from noble metals to refractory metals with increasing resistivity, hardness, melting point and chemical reactivity [4]. Contacts made of pure gold can have low (due to low resistivity and low hardness) and stable (due to low chemical reactivity) resistance, but tend to stick (due to low melting point). On the other hand, refractory contacts can have high (due to high resistivity and high hardness) and varying (due to high chemical reactivity) resistance, but can last many cycles without stiction (due to high melting point). High hardness also requires high contact force, hence, high actuation voltage, which 


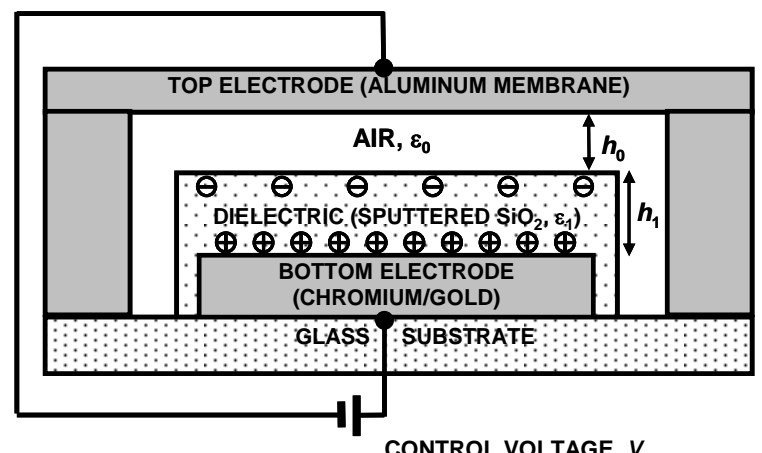

Fig. 1. Schematic cross section of an RF MEMS capacitive switch. Charging of the top and bottom of the dielectric is indicated for the case of a positive control voltage [8].

is not only inconvenient, but also a source of switching noise. Considering the different trade-offs, gold alloys are reasonable starting points for empirical optimization of detailed composition, structure, fabrication process, geometry, contact force, and ambient.

The same failure mechanisms caused by electrical stress also make ohmic switches vulnerable to electrostatic discharging and "hot switching" (with the RF power constantly applied). As low as $100 \mathrm{~V}$ of human-body model was found to produce failure [5]. Hot switching under $10 \mathrm{~mW}$ of RF power can reduce the switch lifetime from billions to millions of cycles [6]. In comparison, capacitive switches usually are immune to electrostatic discharge and can withstand hot switching of approximately $1 \mathrm{~W}$ [7].

\section{FAILURE MECHANISMS OF CAPACITIVE SWITCHES}

Fig. 1 shows that the dielectric insulator of a capacitive switch is usually fixed to one of the two electrodes of the capacitor, while making intermittent contact with the other electrode. When the control voltage, either positive or negative, exceeds the actuation voltage, the switch is closed and charge can be injected into the dielectric from either the fixed or the intermittent contact. Lacking better terminology, the charge injected from the intermittent contact is referred to as "surface charge," while the charge injected from the fixed contact is called "bulk charge." When the control voltage is removed and the intermittent contact is broken, bulk charge can readily return through the fixed contact within seconds, while surface charge must diffuse across the thickness of the dielectric - a process that can take hours [8]. When a control voltage of the same sign is reapplied, surface charge will increase the magnitude of the apparent actuation voltage whereas any remaining bulk charge will decrease the magnitude of the apparent actuation voltage.

Because surface charge is difficult to dissipate, it should be avoided in reliable switches. Unlike bulk

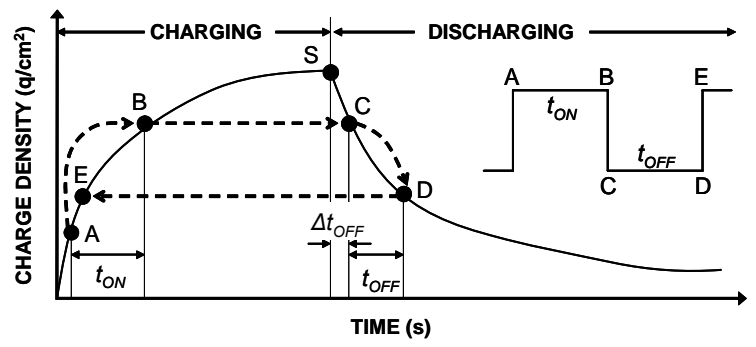

Fig. 2. Dynamic balance of charging (left) and discharging (right) characteristics. After a number of switching cycles, the dielectric accumulates a certain amount of charge as indicated by " $A$ " on the charging curve. During the next on time, charge increases from " $\mathrm{A}$ " to "B." When the switch is off, charge is mapped to " $\mathrm{C}$ " on the discharging curve, which is then discharged to "D." At the beginning of the next switching cycle, charge is mapped from " $\mathrm{D}$ " back to " $\mathrm{E}$ " on the charging curve. Through such a ratchet action, charge is incremented from " $A$ " to "E" after one complete cycle [12].

charging, surface charging is not only determined by the metal contact and dielectric insulator, but also surface and ambient conditions. With proper design and process, the threshold voltage for surface charging can be made higher than the actuation voltage. In this case, the lifetime of the switch is mainly determined by bulk charging, which is simpler to control and characterize than surface charging.

Using a novel femto-ampere transient-current measurement technique, we have extracted the steadystate charge density and charging/discharging time constants of bulk charging in state-of-the-art $\mathrm{Al} / \mathrm{SiO}_{2} / \mathrm{Cr}$ switches [9]. The charge density was found to depend exponentially on voltage and temperature, whereas the time constants remained independent of voltage and temperature [10]. Further, since the charge density never truly reaches a steady state, a series of two or three exponential functions, with increasingly smaller charge density but longer time constants were used to model bulk charging as shown in the following:

$$
Q(t)=\sum_{J=1,2,3} Q_{0 J} \cdot \mathrm{e}^{\frac{V}{V_{0 J}}} \cdot \mathrm{e}^{-\frac{E_{A J}}{k T}} \cdot\left(1-\mathrm{e}^{-\frac{t_{O N}}{\tau_{C J}}}\right) \mathrm{e}^{-\frac{t_{O F F}}{\tau_{D J}}},
$$

where $Q$ is the cumulative charge in the dielectric, $t$ is the cumulative time of operation, $Q_{0}$ is the steady-state charge density, $V$ is the control voltage, $V_{0}$ is the voltage scaling factor, $E_{A}$ is the activation energy, $k$ is the Boltzmann constant, $T$ is the ambient temperature, $t_{O N}$ and $t_{O F F}$ are on and off times of each switching cycle, and $\tau_{C}$ and $\tau_{D}$ are charging and discharging time constants. Higher-order terms can improve the precision of the series, but they are increasingly more difficult to extract. Although stretched exponential functions have been proposed [11], we found the series of simple exponential functions more robust in model extraction and simulation. 
Eq. (1) can be used to evaluate the shift in actuation voltage under different control-voltage waveforms. Fig. 2 shows that, even for a simple square wave of $50 \%$ duty factor, charge accumulation in the dielectric is actually through a dynamic balance of charging and discharging within each switching cycle [12]. Initially, charging is very fast when the switch is on (closed), whereas discharging is very slow when the switch is off (open). After a number of cycles, the accumulated charge retards further charging but accelerates discharging. Therefore, the net incremental charge accumulated after each switching cycle decreases until charging and discharging are exactly balanced within a switching cycle. In this case, the steady-state charge density after dynamic balance is reached is a more important figure of merit than the initial rate of charging [13] in determining the switch lifetime. Similarly, because charging is sensitive to the control-voltage waveform, neither the total number of cycles [2] nor the total amount of contact time [14] is a universal measure of the reliability of a capacitive switch. To compare or predict the lifetime of a capacitive switch under different control-voltage waveforms, steady-state charge densities and charging/discharging time constants must be used.

In addition to space charge, polarization charges of different dimensions and time constants in the dielectric have also been considered [11]. Their effects tend to be short-ranged making them more important to surface charging than bulk charging. For this reason, we prefer to characterize charging through the shift in actuation voltage than the shift in release voltage.

\section{Mitigation OF DiELECTRIC CHARGING}

\section{A. Improved Dielectric Material}

Similar to the gate dielectric of MOSFETs, the dielectric in MEMS capacitive switches must meet a long list of requirements, including dielectric constant, breakdown strength, leakage current, surface roughness, chemical stability, thermal stability, mechanical stability, process ease, reproducibility, etc., in addition to steady-state charge density and charging/discharging time constants. However, because most MEMS processes are limited to relatively low temperatures $\left(\sim 300^{\circ} \mathrm{C}\right)$, the quality and reproducibility of the dielectric used in MEMS are often inferior to that of the MOSFET gate dielectric. Both silicon oxide and silicon nitride, commonly available in MOSFET processes, can be optimized for MEMS capacitive switches. Silicon oxide appears to have a lower charge density but a more reactive surface. By optimizing the silicon oxide process over the last few years, we have reduced its charge density by several orders of magnitude, allowing the

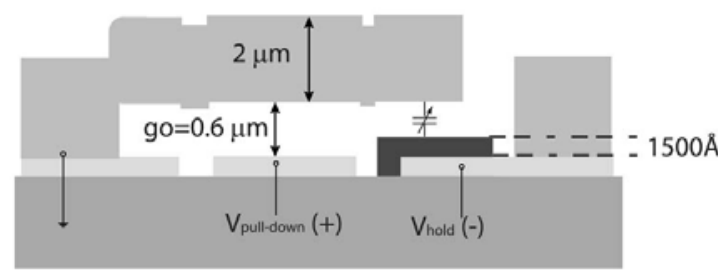

Fig. 3. Cross section of a capacitive switch with $V_{P U L L-D O W N}=$ $30 \mathrm{~V}$ and $V_{H O L D}=-5 \mathrm{~V}$ applied on the actuator and contact separately [18].

switches to be operated for more than 100-billion cycles [2].

\section{B. Reduced Actuation Voltage}

Because of the exponential voltage dependence of the charge density [9], charging can be greatly reduced if lower actuation voltages are required. However, the actuation voltage affects other switch characteristics and careful trade-offs must be made. For example, either a more compliant intermittent contact or a smaller air gap between the intermittent contact and the dielectric can reduce the actuation voltage. But, a more compliant contact tends to stick while a smaller air gap increases the off capacitance. For dielectrics of different thicknesses, charging is actually dependent on the field instead of the voltage. A thicker dielectric reduces the field beneficially but decreases the on capacitance undesirably.

\section{Reduced Dielectric Contact Area}

In general, dielectric charging is proportional to the dielectric contact area. Standoffs of either dielectric [15] or metal [16] have been designed to reduce or eliminate the dielectric contact area. Contact area can also be reduced by increasing the surface roughness with the incorporation of carbon nanoparticles in silicon nitride [17], for example. In most cases, dielectric charging was reduced but other performance characteristics such as contact force or on/off capacitance ratio were compromised. Most recently, a promising design using two control voltages on the actuator and the contact separately was demonstrated (Fig. 3) [18]. The relatively high control voltage $(30 \mathrm{~V})$ on the actuator allows the intermittent contact to be normally suspended at distance with low off capacitance. The relatively low control voltage $(5 \mathrm{~V})$ on the actual contact gives high and reproducible on capacitance. Dielectric charging is eliminated at both the actuator (because no dielectric is used there) and the contact (because the control voltage is relatively low there).

\section{Optimized Control-Voltage Waveforms}

To mitigate dielectric charging, instead of two control voltages on the actuator and contact separately, a highlow control-voltage waveform [19] can also be used 
when the actuator doubles as the contact. This is because the actuation voltage is usually higher than the release voltage. The control voltage needs to be higher than the actuation voltage to close the air gap between the intermittent contact and the dielectric, but can be reduced to only higher than the release voltage to hold the intermittent contact and the dielectric together. If the control voltage is lower than the actuation voltage most of the time, then charging can be reduced even for switches that require a relatively high actuation voltage. Similarly, a bipolar waveform, under which the control voltage alternates between positive and negative after each switching cycle, has been proposed [20] to cancel the charging between the positive and negative cycles. We found [21] that the bipolar waveform reduced charging significantly but did not eliminate it completely, probably because a slight imbalance between positive and negative cycles would allow a small amount of charge, either positive or negative, to be accumulated, which would further upset the balance.

\section{CONCLUSION}

Selected ohmic and capacitive switches have been demonstrated to be reliable. The failure mechanisms of capacitive switches are better understood than that of ohmic switches. There are many ways to mitigate the failure mechanisms of capacitive switches. Models have been developed to predict the lifetime of capacitive switches under accelerated life tests.

\section{ACKNOWLEDGEMENT}

This work was supported in part by the IMPACT Center for Advancement of MEMS/NEMS VLSI, funded by DARPA, through Grant No. HR0011-06-1-0046, under the DARPA N/MEMS Science \& Technology Fundamentals Research Program.

\section{REFERENCES}

[1] J. Maciel, S. Majumder, R. Morrison, and J. Lampen, "Lifetime characteristics of ohmic MEMS switches," Proc. Int. Soc. Optical Engineering, vol. 5443, pp. 9-14, 2004.

[2] C. L. Goldsmith, D. I. Forehand, Z. Peng, J. C. M. Hwang, and J. L. Ebel, "High-cycle life testing of RF MEMS switches," IEEE MTT-S Int. Microwave Symp. Dig., pp. 1805-1808, June 2007.

[3] P. G. Slade, Ed., Electrical Contacts: Principles and Applications, CRC Press, 1999.

[4] Q. Ma, Q. Tran, T. A. Chou, J. Heck, H. Bar, R. Kant, and V. Rao, "Metal contact reliability of RF MEMS switches," Proc. Int. Soc. Optical Engineering, vol. 6463, p. $646305,2007$.

[5] J. A. Warren et al, "Human body model, machine model and charged device model ESD testing of surface micromachined microelectromechanical systems (MEMS)," Dig. EOS/ESD Symp., pp. 238-248, 2001.
[6] S. Majumder, J. Lampen, R. Morrison, and J. Maciel, “A packaged, high-lifetime ohmic MEMS RF switch," IEEE MTT-S Int. Microwave Symp. Dig., pp. 1935-1938, June 2003.

[7] B. Pillans, J. Kleber, C. Goldsmith, and M. Eberly, "RF power handling of capacitive RF MEMS devices," IEEE MTT-S Int. Microwave Symp. Dig., pp. 329-332, June 2002.

[8] Z. Peng, X Yuan, J. C. M. Hwang, D. Forehand, and C. L. Goldsmith, "Top vs. bottom charging of the dielectric in RF MEMS capacitive switches," Proc. Asia-Pacific Microwave Symp., Dec. 2006.

[9] X. Yuan, J. C. M. Hwang, D. Forehand, and C. L. Goldsmith, "Modeling and characterization of dielectriccharging effects in RF MEMS capacitive switches," IEEE MTT-S Int. Microwave Symp. Dig., pp. 753-756, June 2005.

[10] X. Yuan, Z. Peng, J. C. M. Hwang, D. Forehand, and C. L. Goldsmith, "Temperature acceleration of dielectric charging in RF MEMS capacitive switches," IEEE MTTS Int. Microwave Symp. Dig., pp. 47-50, June 2007.

[11] G. I. Papaioannou, M. Exarchos, V. Theonas, G. Wang, and J. Papapolymerou, "On the dielectric polarization effects in capacitive RF-MEMS switches," IEEE MTT-S Int. Microwave Symp. Dig., pp. 761-764, June 2005.

[12] X. Yuan, Z. Peng, J. C. M. Hwang, D. Forehand, and C. L. Goldsmith, "A transient SPICE model for dielectriccharging effects in RF MEMS capacitive switches," IEEE Trans. Electron Devices, vol. 53, pp. 2640-2648, Oct. 2006.

[13] S. Melle, D. De Conto, D. Dubuc, K. Grenier, O. Vendier, J-L. Muraro, J-L. Cazaux, and R. Plana, "Reliability modelling of capacitive RF MEMS," IEEE Trans. Microwave Theory Techniques, vol. 53, pp. 34823488, Nov. 2005.

[14] W. M. Van Spengen, R. Puers, R. Metens, and I. De Wolf, "Experimental characterization of stiction due to charging in RF MEMS," Dig. Int. Electron Devices Meet., pp. 901-904, Dec. 2002.

[15] C. L. Goldsmith, "Proximity micromechanical systems," United States Patent 6,608,268, Aug. 2003.

[16] P. Blondy, A. Crunteanu, C. Champeaux, A. Catherinot, P. Tristant, O. Vendier, J. L. Cazaux, and L. Marchand, "Dielectric less capacitive MEMS switches," IEEE MTTS Int. Microwave Symp. Dig., pp. 573-576, June 2004.

[17] C. Bordas, K. Grenier, D. Dubuc, E. Flahaut, S. Pacchini, M. Paillard, and J-L. Cazaux, "Carbon nanotube based dielectric for enhanced RF MEMS reliability," IEEE MTT-S Int. Microwave Symp. Dig., pp. 375-378, June 2007.

[18] B. Lakshminarayanan, and G. Rebez, "High-power highreliability sub-microsecond RF MEMS switches capacitors," IEEE MTT-S Int. Microwave Symp. Dig., pp. 1801-1804, June 2007.

[19] C. L. Goldsmith, J. Ehmke, A. Malczewski, B. Pillans, S. Eshelman, Z. Yao, J. Brank, and M. Eberly, "Lifetime characterization of capacitive RF MEMS switches," IEEE MTT-S Int. Microwave Symp. Dig., pp. 1227-230, June 2001

[20] J. R. Reid, and R. T. Webster, "Measurements of charging in capacitive microelectromechanical switches," Electron. Lett., vol. 38, pp. 1544-1545, Nov. 2002.

[21] Z. Peng, X. Yuan, J. C. M. Hwang, D. Forehand, and C. L. Goldsmith, "Dielectric charging of RF MEMS capacitive switches under bipolar control-voltage waveforms," IEEE MTT-S Int. Microwave Symp. Dig., pp. 1817-1820, June 2007. 